\title{
Towards a best-practices guide for camera trapping: assessing differences among camera trap models and settings under field conditions
}

\author{
P. Palencia ${ }^{1}$ (D) J. Vicente ${ }^{1}$ (D) R. C. Soriguer ${ }^{2}$ (D) \& P. Acevedo ${ }^{1}$ \\ ${ }^{1}$ Instituto de Investigación en Recursos Cinegéticos (IREC) CSIC-UCLM-JCCM, Ciudad Real, Spain \\ ${ }^{2}$ Estación Biológica de Doñana (EBD-CSIC), Sevilla, Spain
}

\section{Keywords}

camera trap performance; encounter rate; imperfect detection; probability of detection; remote cameras; trigger speed; wildlife monitoring.

\section{Correspondence \\ Pablo Palencia and Pelayo Acevedo, Instituto de Investigación en Recursos Cinegéticos, IREC (UCLM-CSIC-JCCM), Ronda de Toledo 12, 13071, Ciudad Real, Spain. Pablo Palencia: Tel: 34926295300 \\ Email: palencia.pablo.m@gmail.com (PP); pacevedo@irec.csic.es (PA)}

Editor: Matthew Hayward

Received 12 July 2021; revised 3 November 2021; accepted 4 November 2021

doi:10.1111/jzo.12945

\begin{abstract}
Camera trapping is a widely used tool in wildlife research and conservation, and a plethora of makes and models of camera traps have emerged. However, insufficient attention has been paid to testing their performance, particularly under field conditions. In this study, we have comparatively tested five of the most frequently used makes of camera trap (Bushnell, KeepGuard, Ltl Acorn, Reconyx and Scoutguard) to identify the key factors behind their probability of detection (i.e. the probability that the camera successfully capturing a usable photograph of an animal passing through the field of view) and trigger speed (i.e. the time delay between the instant at which a motion is detected, and the time at which the picture is taken). We used 45 cameras (nine devices of each make) with infrared flash in a field experiment in which a continuous remote video was used in parallel (as a gold-standard) to discover the animals that entered the camera trap detection zone. The period (day/ night), distance between animals and cameras, model, species, deployment height and activation sensitivity were significantly related to the probability of detection. This probability was lower during the night than during the day. There was a greater probability of detecting a given species when the cameras were set at its shoulder height. The interaction between species and the distance between the animals and the cameras significantly affected the trigger speed, meaning that the closer the animals that entered the detection zone, the higher the trigger speed, with substantial differences among species. This was probably related by movement speed. In conclusion, this study shows differences in the performance of camera trap models and settings, signifying that caution is required when making direct comparisons among results obtained in different experiments, or when designing new ones. These results provide empirical guidelines for best practices in camera trapping and highlight the relevance of field experiments for testing the performance of the camera traps.
\end{abstract}

\section{Introduction}

The use of autonomously triggered cameras (camera traps) for wildlife research has exploded since 2000 (Burton et al., 2015). Camera trapping is widely used extensively in terrestrial vertebrate research for a wide range of purposes, including species inventories (Tobler et al., 2008), abundance and density estimates (Gilbert et al., 2021), and movement and behaviour studies (Caravaggi et al., 2017; Niedballa et al., 2019). Camera trapping can be carried out at a relatively low cost (barring the initial purchase), it is minimally invasive, and makes it possible to monitor elusive species and/or remote areas (Rovero \& Zimmermann, 2016).

The wide range of applications has led to the emergence of a plethora of camera trap makes and models during the last few years. These makes differ as regards their features, specifications and costs (Rovero \& Zimmermann, 2016). This wide range of camera traps signifies that a rigorous way in which to test their performance is required to integrate the data from different experiences and thus to carry out effective wildlife management and conservation programmes, since there are still several issues regarding the use of camera traps (Delisle et al., 2021; Glover-Kapfer et al., 2019). Some studies have indicated the malfunctioning of camera traps and the high variability in performance among makes and devices in terms of missed detections (Hofmeester et al., 2019; Jacobs \& Ausband, 2018; McIntyre et al., 2020). For instance, some authors have described factors affecting the performance of cameras, such as (1) environmental conditions, that is, humidity or temperature (Jacobs \& Ausband, 2018; Kays et al., 2010; McIntyre et al., 
2020; Rowcliffe et al., 2011); (2) target species, owing to body size (Hofmeester et al., 2017) and speed of movement (Rowcliffe et al., 2011) and (3) camera trap deployment and settings, that is, height and sensitivity (Swann et al., 2004). Missed detections could potentially generate misleading results in camera trapping studies, and therefore, create difficulties as regards direct comparison among studies (Jacobs \& Ausband, 2018). In this respect, despite some statistical approaches accounting for the imperfect detection (Guillera-Arroita et al., 2010), recent studies have shown that biased and imprecise results can be obtained when detection probabilities are low (Hofmeester et al., 2021; Kays et al., 2021). Additionally, (1) there are a wide variety of studies that used the raw encounter rates without accounting for false-negatives (i.e. those animals/ sequences that were not recorded by the camera trap but actually entered the area monitored by the camera, the field of view - FOV). For instance, those focused on species interactions, activity patterns, or inventories, among others and (2) some of the methods that account for imperfect detection assumed certain detectability at some point of the detection zone (e.g. Howe et al., 2017), which could not be always realistic. These reasons signify that assessing the performance of camera traps under field conditions, along with defining limitations and applications, still requires further research (Meek et al., 2015). Understanding the constraints of camera trapping in a broad sense, and for a range of camera trap models, will assist researchers to (1) make decisions about which camera trap and settings are appropriate for a given study; (2) compare the results of studies that use different camera trap models and (3) include uncertainty regarding camera trap detection in subsequent modelling approaches.

However, testing camera traps under field conditions is challenging because it requires a high sampling effort in terms of the time and number of devices required to achieve a useful sample size. It is for this reason that most comparisons have been carried out in laboratory trials and using humans, or even domestic animals, walking in front of the camera traps (Apps \& McNutt, 2018; Cusack et al., 2015; Seccombe, 2017; Swann et al., 2004; Yajima \& Nakashima, 2021). When wild animals are eventually considered as the target, other difficulties emerge, which are mainly related to false-negatives (Driessen et al., 2017; Hughson, Darby, \& Dungan, 2010; Jacobs \& Ausband, 2018). Undetected sequences are never quantified, and may therefore lead to an important overestimation of the performance of cameras. For an appropriate validation of the performance of a camera trap, it is mandatory to know the number of opportunities that had to record animals. The assessment, therefore, ideally requires the recording of all the animals that enter the FOV of the camera traps, using an independent device and/or approach.

In this context, the objective of this study was to gain better understanding of the performance of camera traps and to evaluate the key factors that influence their probability of detection (i.e. the probability that the camera successfully capturing a usable photograph of an animal passing through the FOV) and trigger speed (i.e. the time delay between the instant at which a motion is detected, and the time at which the picture is taken). In camera trapping studies, probability of detection is usually described as a combination of three conditional probabilities. First, the probability that an animal moves in front of the camera; second, the probability that the animal triggers the PIR; and third, the probability that the animal is identifiably detected on an image (Findlay et al., 2020; Hofmeester et al., 2019). Thus, we can separate into availability (first component, was the animal in the area) and detectability (second and third components, did the camera work as expected). In this study, we were focused on the detectability component. Probability of detection and trigger speed are the most frequently demanded technological developments and relate to sensor performance (Glover-Kapfer et al., 2019; Meek \& Pittet, 2012). We have therefore tested five different camera trapping models (of five makes: Bushnell, KeepGuard, LtlAcorn, Reconyx and Scoutguard), different settings (sensitivities and period of the day) and deployment characteristics (height) in a field experiment in which wild animals were considered to be the target. We monitored the presence of animals in the FOV of cameras and the adjacent area using an external video camera $24 / 7$ as a goldstandard reference for all the animals that should actually have been recorded by the camera traps.

\section{Material and methods}

\section{Study area}

The study was carried out in the ICTS (Scientific Technical Infrastructure) of the Doñana Biological Reserve (ICTS-RBD, http://icts.ebd.csic.es/es/inicio), a territory of approximately 6800 ha located in Doñana National Park $\left(37^{\circ} 0\right.$ 'N, $6^{\circ} 30^{\prime} \mathrm{W}$; DNP). Doñana National Park is one of the most important nature reserves in Europe in terms of biodiversity and was declared a Biosphere Reserve by UNESCO in 1980. The Doñana Biological Reserve is located on the Atlantic coast of south-west Spain and is dominated by Mediterranean shrubland and characterised by seasonal marshland.

\section{Field experiment}

\section{Sampling period}

The experiment was carried out during a 5-week period in the summer of 2018 (July 13rd to August 19th). The weather during the survey was hot and dry: the average daily temperature was $29.93^{\circ} \mathrm{C}$, the average temperature at sunrise was $18.40^{\circ} \mathrm{C}$, the average maximum temperature was $32.12^{\circ} \mathrm{C}$, and there was no rainfall throughout the survey (data from a weather station located $4 \mathrm{~km}$ away from the experiment). These values are within the range of operating environmental weather conditions described by the manufacturers (Table 1).

\section{Experimental design}

The experiment took place in the ecotone between marshland and shrubland, close to a natural water hole (Fig. 1). This location was selected so as to detect a high number of species and animals during the survey, since water and grassland are highly demanded resources in the study area during the 
Table 1 Key technical specifications of the camera trap models tested in the study. All the parameters included in this table were obtained from the official user manuals, with the exception of the FOV of Reconyx, which was obtained from https://www.trailcampro.com/products/reconyxhc500-review\#tab2, since it was not included in the user manual

\begin{tabular}{|c|c|c|c|c|c|}
\hline & BUS & KPG & LAC & RNY & SCT \\
\hline Lens FOV $\left(^{\circ}\right)$ & $42 / 45^{a}$ & 50 & 55 & 42 & 40 \\
\hline IR - Flash Range (m) & 30 & $12-15$ & 20 & 15.25 & $10-12$ \\
\hline Trigger time (s) & 0.2 & 1 & 0.6 & 0.25 & 1.2 \\
\hline Trigger interval (s) & 1 & 5 & 0 & RapidFire $^{\mathrm{b}}$ & 1 \\
\hline Operating temperature $\left({ }^{\circ} \mathrm{C}\right)$ & -20 to 60 & -20 to 60 & -30 to 70 & -7 and above $e^{c}$ & -20 to 60 \\
\hline Storage temperature $\left({ }^{\circ} \mathrm{C}\right)$ & -30 to 70 & -30 to 70 & NA & NA & -30 to 70 \\
\hline Operating humidity (\%) & 5 to 90 & 5 to 90 & 5 to 95 & NA & 5 to 90 \\
\hline $\operatorname{Cost}^{d}$ & $€ €$ & $€$ & $€$ & $€ € €$ & $€$ \\
\hline
\end{tabular}

Camera trap model abbreviations: BUS, Bushnell Trophy Cam Aggressor model 119774; FOV, field of view; KPG, KeepGuard 680V; LAC, Ltl Acorn Ltl-5310 Series; RNY, Reconyx HyperFire HC500; SCT, Scoutguard SG550BV. All the cameras were purchased in 2017.

$42^{\circ}$ in French, Spanish, German and Italian sections, and $45^{\circ}$ in English section of the same manual.

'The manual indicated as far option 'RapidFire'.

'Using AA Alkaline batteries.

${ }^{\mathrm{I}} €$ (price ranged from 100 to $170 €$ ), €€ (price ranged from 220 to $280 €$ ), and $€ € €$ (price ranged from 350 to $400 €$ ).

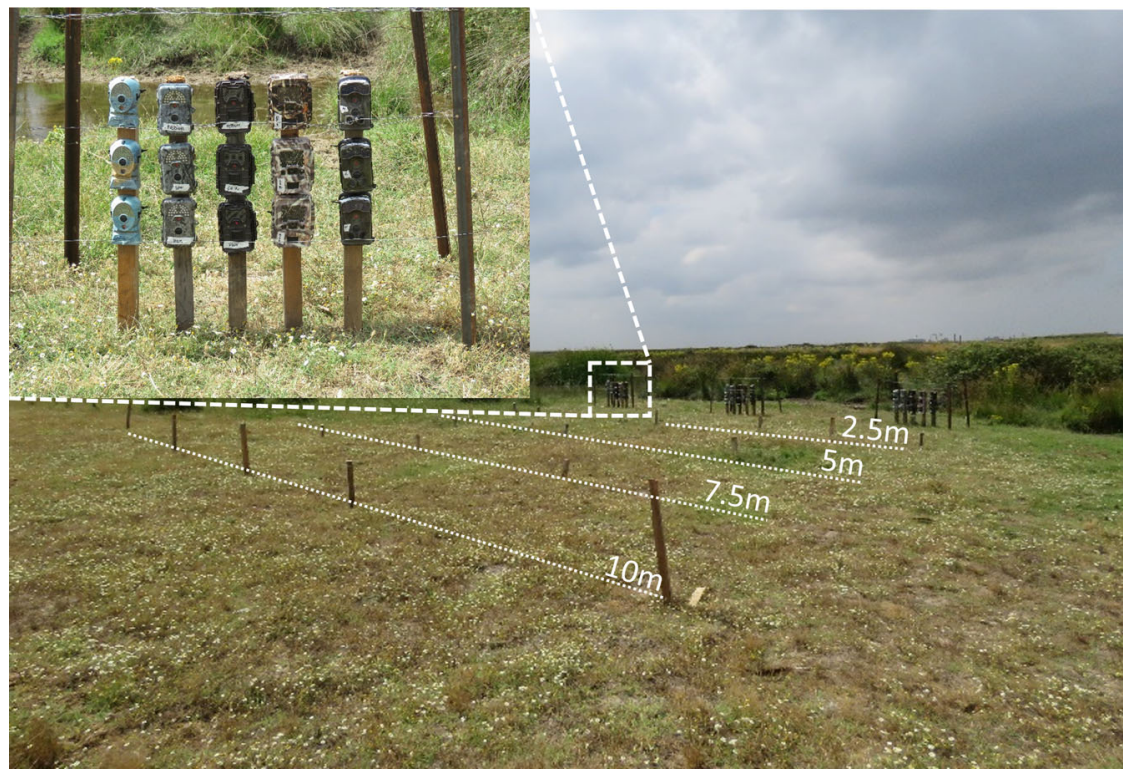

Figure 1 Experimental design used in this study. Note the three sampling points, and the wooden sticks defining the distance intervals. The top left-hand corner contains a photo of one of the sampling points.

summer (Barasona et al., 2014; Barroso et al., 2020). The vegetation in front of the cameras was short grass (Fig. 1) and there was no slope.

We tested forty-five camera traps of five different models (corresponding to five different makes, and nine of each device): Bushnell Trophy Cam Aggressor model 119774 (BUS), Keep Guard 680V (KPG), Ltl Acorn Ltl-5310 Series (LAC), Scoutguard SG550BV (SCT), and Reconyx HyperFire HC500 (RNY) (Table 1); all of them used passive infrared motion sensors (PIR) and infrared flash. All the camera traps were programmed to be operative all day, and to record one photo in each activation with the minimum time lapse between consecutive activations. The date and time were automatically stamped onto each photo. We checked the camera traps twice to change their batteries and memory cards, when necessary, and to ensure that they were functioning correctly. We used the same alkaline batteries in all the camera traps.

We designed an experiment with three spatial replicates (sampling points; Fig. 1). At each sampling point, three camera traps of the same model were placed on the same stick at three different heights from the ground $(30,45$ and $60 \mathrm{~cm})$. Camera traps of different models were placed side by side, no more than $10 \mathrm{~cm}$ apart, and aligned vertically and horizontally. Three cameras of each model were set to a low, medium and high sensitivity, and the height at which cameras with a given sensitivity configuration was rotated between sampling points. 
Wooden sticks were placed to define four $2.5 \mathrm{~m}$ wide intervals, from 0 to $10 \mathrm{~m}$, within the FOV of the camera traps (Fig. 1). These sticks were used to delimitate the FOV of each sampling point (i.e. a common detection zone for the 15 camera traps at each sampling point) and were later used to locate the position of the individuals registered. We considered $10 \mathrm{~m}$ as maximum distance based on previous studies that reported detection distances for the species sampled in this study (Hofmeester et al., 2017; Palencia, Rowcliffe et al., 2021). The cameras were angled to be parallel to the slope of the ground. This design probably modified the basal encounter rate and behaviour of the animals, but did not introduce bias into our experiment and analysis, as we focused on those animals that entered the FOV and we did not consider their behaviour.

The gold-standard reference for the animals entering the FOV of the camera traps consisted of monitoring the sampled area and surroundings with a video camera that recorded and streamed in a continuous mode (infrared at night) throughout the study period (Appendix S1). This camera was supported by the ICTS-RBD. For each sampling point, we only considered those animals that crossed the centre of the FOV of the central camera trap. We considered this criterion to discard those animals that entered and left on the edge of the FOV of external cameras and could not be potentially recorded for all of them.

\section{Data processing}

\section{Video camera}

For those animals that entered the FOV at each sampling point, we annotated: the date and time of each sequence, the species and the distance interval of the first animal that entered the FOV. We considered as a sequence every animal or group of animals that entered the FOV. When more than one animal entered the FOV simultaneously, that which was closest to the camera traps was considered to estimate the distance interval parameter.

\section{Camera traps}

Camera trapping may generate a high amount of data without information, 'blank-images' or 'false-positives', because of vegetation, the sun or dappled shade activating the cameras (Glover-Kapfer et al., 2019). All the images recorded were first assessed for the presence of animals, after which they were annotated for each sequence: the date and time, the species and the distance interval of the first and closest animal in the sequence.

\section{Analysis}

We first compared the total number of photos (including blank images) recorded by each camera trap as regards sensitivity settings and models. Subsequent analyses were carried out with the information concerning the sequences of animals entering the FOV at a distance closer than $10 \mathrm{~m}$. Two generalised linear mixed models (GLMM) with a binomial distribution and logit link function were used to assess the performance of the camera traps as regards the probability of detection and trigger speed, respectively. When modelling the probability of detection, our response variable was the detection of each sequence recorded by the video camera ( 1 recorded by a camera trap, 0 unrecorded). When modelling trigger speed considering only the captures obtained by the camera traps, the response variable was 1 when the camera trap recorded the animal in the same distance interval that it entered, and 0 when it was recorded in another interval. The explanatory variables used in both models were species group (fixed factor, with seven levels for detection probability model like carnivores, big birds, horses, herons, small birds, medium-sized birds and wild boar and five levels for trigger speed. See details in Appendix S2), height (fixed factor, three levels: 30,45 and $60 \mathrm{~cm}$ ), camera trap model (fixed factor, five levels: BUS, KPG, LAC, RNY and SCT), sensitivity (fixed factor, three levels: low, moderate, high), period (fixed factor, two levels: day and night), sampling point (fixed factor, three levels), and distance interval (fixed factor, four levels, one every $2.5 \mathrm{~m}$ up to $10 \mathrm{~m}$ ) at which the animal entered the FOV. With regard to the period, we considered night sequences to be those in which the IR flash was activated. In the probability of detection model, we also tested the interactions between height deployment and species group, camera trap model and species group, camera trap model and period, camera trap model and sensitivity, and height and distance interval. In the model for the trigger speed, we also tested the interaction between distance interval and species group. Since body size has been described as being one of the most important factors in camera trap detections (Hofmeester et al., 2017), we followed Driessen et al. (2017) and pooled species of similar body size in groups for analytical purposes. Details on the grouping of species included are provided in Table 1, Appendix S2. The sequence was included in both models as a random effect factor and the sampling point (three levels) as a fixed factor (Harrison et al., 2018). A backward stepwise procedure based on Akaike's Information Criteria was applied to select the most parsimonious models (Akaike, 1974). Post hoc comparisons for categorical factors were performed using Tukey's method. The models were validated according to the exploration of residuals and following to Zuur et al. (2010). The level of statistical signification was established at $P<0.05$.

\section{Results}

The video camera recorded 3788 sequences of 27 different species, including reptiles, rodents, birds (including a high number of taxonomic families), lagomorphs, carnivores, and wild and domestic ungulates. The most frequent group was that of birds (2963 sequences), followed by mammals (819) and reptiles (4). The most frequently recorded species was the magpie (Pica pica), with 1923 sequences.

With regard to the camera traps, we recorded 270530 photos that included 1083 independent sequences of animals that entered any FOV at a shorter distance than $10 \mathrm{~m}$ from the camera traps. We recorded 27 different species (see details in Appendix S2), and we did not discard any sequence due to 


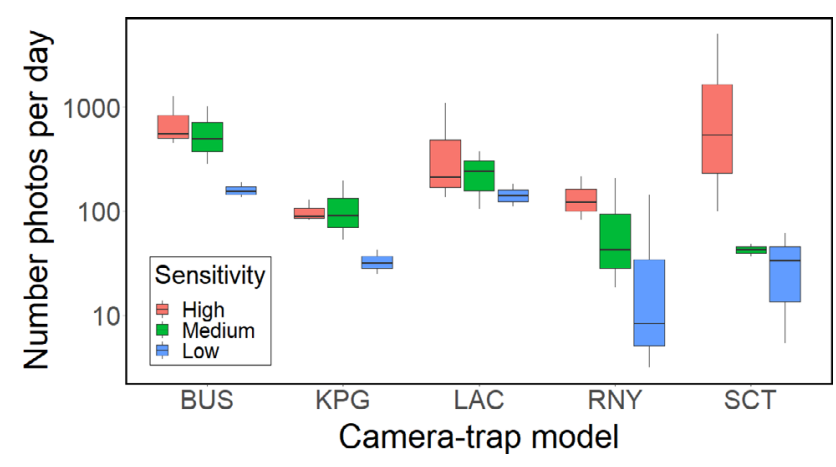

Figure 2 Box-plot of the average number of photos recorded per day by each camera trap model and sensitivity. Camera trap model abbreviations: BUS, Bushnell Trophy Cam Aggressor model 119774; KPG, KeepGuard 680V; LAC, Ltl Acorn Ltl-5310 Series; RNY, Reconyx HyperFire HC500; SCT, Scoutguard SG550BV.

problems to identify the species due to under- or over-exposed pictures. The minimum number of photos per day (including blank images) was obtained at low sensitivity. This pattern was consistent for all the camera trap models, but there were differences among them as regards the mean number of photos (Fig. 2).

With regard to the GLMM models, we discarded the groups of raptors, hare, deer, rodents and snakes from the analysis owing to the low sample size (i.e. number of sequences; see Appendix S2). When modelling trigger speed, we also discarded herons and small birds because of the low number of sequences of these groups detected by the camera traps. The best model for the probability of detection included the interactions between the camera trap model and period, the camera trap model and sensitivity, the camera trap model and species group, the species group and height and the distance interval and height (Table 2 and Appendix S3). The marginal coefficient of determination $\left(R^{2} \mathrm{~m}\right)$ calculated by theoretical method was 0.70 . Detailed statistical results of this model are provided in Appendix S3.

In the case of the camera trap models, the probability of detection was significantly higher during the day than at night for all models, and significantly different for three of them (BUS, LAC and SCT; Fig. 3a; Appendix S3). We also found significant differences in the probability of detection as regards the sensitivity levels for all the models (a higher probability of detection was achieved at high sensitivity). In some models (e.g. RNY), the predicted probability of detection differed as regards sensitivity levels, but in others (e.g. LAC and BUS), these differences were minimal. In summary, the highest probability of detection was obtained for BUS, which significantly differed from the other camera trap models, with the exception of LAC. The SCT achieved the lowest probability of detection, and was significantly different from BUS and LAC (Appendix S3). It should be noted that the time recorded and stamped by all the BUS devices ran progressively more and more slowly throughout the study period (Appendix S4). We noticed this during image processing, and corrected this time delay, as multiple camera traps were placed at the same point, and we therefore had multiple records of the same animals.

With regard to the species groups, the highest predicted probability of detection was obtained for wild boar and carnivores $(>0.9)$, while the lowest was obtained for small and medium-sized birds $(<0.4$, Fig. 3b-d; Appendix S3). Wild boar obtained a significantly higher predicted probability of detection than other species groups. Our results show that LAC achieved the highest probability of detection for all the bird groups, while BUS achieved the highest for carnivores and wild boar (Fig. 3b-d; Appendix S3). We also evidenced the relevance of camera trap deployment height, with significant effects on some species groups (e.g. herons), signifying that the maximum probability of detection was obtained at the minimum height deployment. With regard to the interaction between camera trap deployment height and distance interval, we found that, at shorter distances, the maximum probability of detection was obtained at a lower height, while as the distance between the animals and the camera traps increased, the highest probability of detection was obtained at higher heights.

Finally, in the case of the trigger speed, we obtained two best candidate models. The simplest one included species group, camera trap model, sampling point, distance interval and the interactions between species group and camera trap model, as well as species group and distance interval (Table 2 and Appendix S3). This model has an $R^{2} \mathrm{~m}$ of 0.26 and its statistical parameters are provided in Appendix S3. Upon considering the interaction between species group and distance interval, the closer the animal entered the FOV, the higher the probability of a fast activation (i.e. the animal was recorded in the distance interval at which it actually entered the FOV) for all the species groups, except the carnivores (Fig. 4). In the case of the carnivores, the expected probability of fast activation was close to 0.5 at all distance intervals. With regard to the interaction between species group and camera trap model,

Table 2 Candidate models explaining probability of detection and trigger speed, respectively

\begin{tabular}{lll}
\hline Candidate models & AlC & Response variable \\
\hline SpG + Hei + CTM + Sen + Per + Poi + Int + SpG*Hei + SpG*CTM + CTM*Per + CTM*Sen + Int*Hei & 9867.7 & Probability of detection \\
SpG + CTM + Poi + Int + CTM*SpG + SpG*Int & 2978.0 & Trigger speed \\
SpG + CTM + Poi + Int + Hei + CTM*SpG + SpG*Int & 2979.4 & Trigger speed \\
\hline
\end{tabular}

All models include ID sequence as a random effect factor.

Abbreviations: CTM, camera trap model; Hei, camera trap deployment height; Int, distance interval; Per, period; Poi, sampling point; Sen, sensitivity; SpG, species group. 

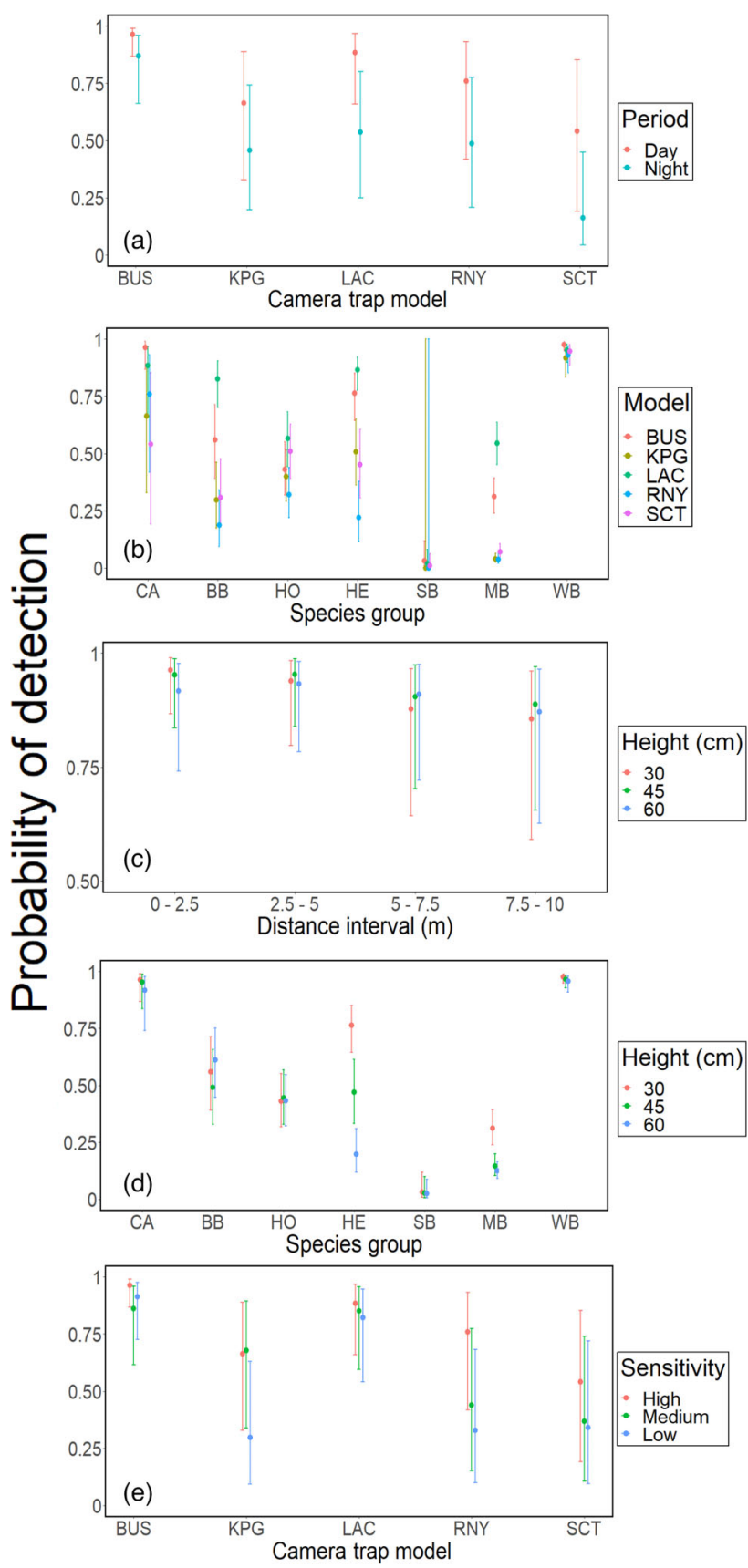

Figure 3 Predicted values for probability of detection of the GLMM model (see Table 2) regarding the interactions between (a) period and camera trap model, (b) species group and camera trap model, (c) height deployment and distance interval of detection, (d) height deployment and species group and (e) sensitivity and camera trap model. Camera trap model abbreviations: BUS, Bushnell Trophy Cam Aggressor; KPG, KeepGuard 680V; LAC, Ltl Acorn Ltl-5310 Series; RNY, Reconyx HyperFire HC500; SCT, Scoutguard SG550BV. Species group abbreviations: CA, carnivores; $\mathrm{BB}$, big birds; $\mathrm{HO}$, horses; $\mathrm{HE}$, herons; SB, small birds; $\mathrm{MB}$, medium birds; WB, wild boars. Error bars are standard errors.

big birds obtained the highest probability of fast activation, and carnivores the lowest for all the models; but there were differences among the models as regards horses, medium-sized birds and wild boar (Fig. 4a).

\section{Discussion}

In this study, we tested five models of some of the most frequently used makes of camera trap to include a range of 


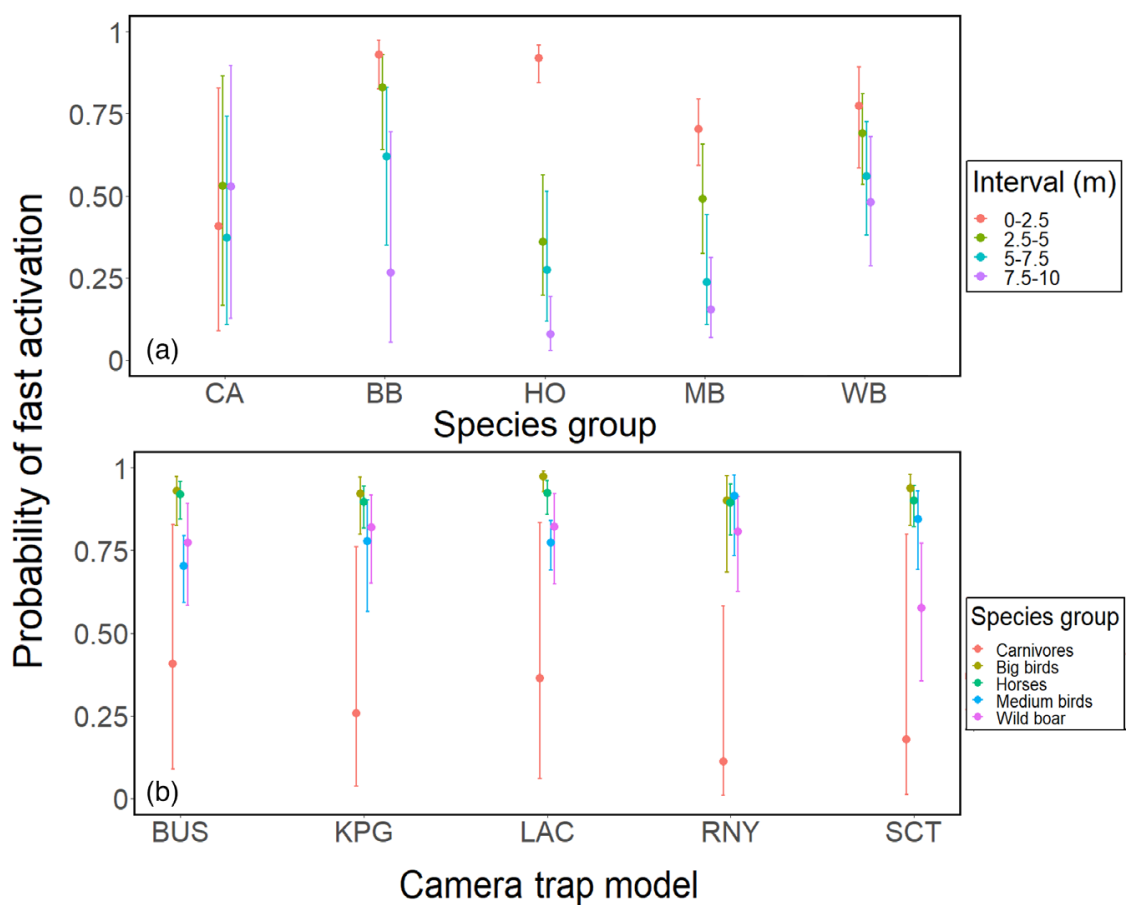

Figure 4 Predicted values for the trigger speed GLMM model (Table 2) in relation to the interactions between (a) species group and distance interval and (b) species group and camera trap model. The probability of fast activation represents the probability of recording an animal at the distance interval in which it actually entered the field of view of the camera trap. Camera trap model abbreviations: BUS, Bushnell Trophy Cam Aggressor; KPG, KeepGuard 680V; LAC, Ltl Acorn Ltl-5310 Series; RNY, Reconyx HyperFire HC500; SCT, Scoutguard SG550BV. Species group abbreviations: $\mathrm{CA}$, carnivores; $\mathrm{BB}$, big birds; $\mathrm{HO}$, horses; $\mathrm{HE}$, herons; $\mathrm{SB}$, small birds; $\mathrm{MB}$, medium birds; WB, wild boars. Error bars are standard errors.

models and features that are indicative of what is on the market (there are dozens of models and several for each make). We demonstrated that false-negatives can lead to substantial data loss, and we identified the main factors that influence the probability of detection (i.e. the probability of the camera successfully capturing a usable photograph of an animal passing through the FOV) and trigger speed (i.e. the time delay between the instant at which a motion is detected, and the time at which the picture is taken) of each one under our study conditions. Overall, our results provide empirical guidelines for best practices in camera trapping research, thus enhancing the applicability, robustness and reliability of camera trapping studies (Fig. 5).

It is frequently stated that false triggers (i.e. blank images) drain battery life and fill memory card space, in addition to increasing the amount of time required for image processing (Rovero \& Zimmermann, 2016). Some authors have pointed out that higher and angled placements, along with higher temperatures, generate more blank images (Jacobs \& Ausband, 2018). Our results confirm that the number of blank images is higher when the sensitivity of the camera traps is higher (Fig. 2; Welbourne et al., 2016). However, to the best of our knowledge, there are no previous studies evaluating this relationship. Some studies have found a correlation between the number of detections of animals and the number of blanks (Driessen et al., 2017). In this study, we have obtained the highest probability of detection with high sensitivity for all the camera traps tested, with the exception of KPG (Fig. 4e). These results can be explained by the fact that at high sensitivity, camera traps are constantly activated by vegetation (or other variables), and the probability of 'randomly' recording animals is, therefore, higher; but this constrains the life of the batteries and memories. Although a high probability of detection is always desired, high sensitivity will suppose a trade-off between detection and memory and battery limitations (Fig. 5). In this respect, the hot and dry scenario in which the study was carried out should be highlighted, which could influence the performance of the cameras.

We also found a significant interaction between the period of the day and the camera trap model with which to explain the probability of detection (Fig. 3a). This probability was lower at night than during the day, which is consistent with the previous studies (Rowcliffe et al., 2014). The magnitude of differences varied among models. Broadly, this is highly relevant for camera trapping studies based on encounter rates (Mills et al., 2020) and those focused on activity patterns (i.e. proportion of the day that animals spend moving), key parameters with which to estimate population density (Palencia et al., 2021a), species interactions (Niedballa et al., 2019) or even when they are the target parameter themselves (Distiller et al., 2020; Edwards et al., 2020). We conclude that the effect of the period of the day should be considered when analysing camera 


\begin{tabular}{|c|c|c|}
\hline Key factors & Practical limitations & Practical recommendations \\
\hline Sensitivity & $\begin{array}{l}\text { Trade-off between detection sensitivity and } \\
\text { memory and battery capacities because of } \\
\text { blank-images }\end{array}$ & $\begin{array}{l}\text { Set high sensitivity anly when memory } \\
\text { and battery can be checked frequently, } \\
\text { and/ar when apen areas are sampled }\end{array}$ \\
\hline Day & $\begin{array}{l}\text { Different probability of detection between } \\
\text { day and night time }\end{array}$ & $\begin{array}{l}\text { Include this variability in the madels when } \\
\text { analysing daily parameters (e.g. activity) }\end{array}$ \\
\hline Madels & $\begin{array}{l}\text { Different probability of detection according to } \\
\text { madels (makes) }\end{array}$ & $\begin{array}{l}\text { Avoid using different camera trap } \\
\text { models in the same study }\end{array}$ \\
\hline & $\begin{array}{l}\text { Bias in prabability of detection when camera } \\
\text { traps are not set at shoulder height of } \\
\text { target species }\end{array}$ & $\begin{array}{l}\text { Manitar exclusively marpholagically similar } \\
\text { species, consider specific sampling design } \\
\text { for each species, ar place more than one } \\
\text { camera at different heights }\end{array}$ \\
\hline Trigger speed & $\begin{array}{c}\text { Slower activations as more distant animals } \\
\text { enters FaV }\end{array}$ & $\begin{array}{l}\text { Use lure to increase the amount of } \\
\text { time in front of the FGV (if it daes not } \\
\text { violate method assumptions) }\end{array}$ \\
\hline
\end{tabular}

Figure 5 Limitations and recommendations obtained in this study as regards key camera trapping factors. This could be considered as bestpractice guide when working with camera traps. Abbreviations: FOV, field of view (area captured by a camera trap).

trapping data to avoid biased results (Fig. 5) by, for instance, weighting the encounter rates (Rowcliffe et al., 2014).

As has occurred in previous studies, we found that the probability of detection varied among camera trap models (Fig. 3). Camera traps are sometimes classified as 'recreational' (e.g. Bushnell TrophyCam) versus 'professional' (e.g. Reconyx Hyperfire PC800) on the basis of technical specifications and price (Newey et al., 2015). However, upon considering the results reported both here and in previous studies (Apps \& McNutt, 2018), these categories do not always correspond to performance. Some cheaper camera trap models can perform just as well as or even better than more expensive ones (Driessen et al., 2017). In this respect, the published studies are not consistent. For instance, some studies have indicated the poor performance of Reconyx when compared with Little Acorn and Bushnell (Apps \& McNutt, 2018; Fancourt et al., 2018; Seccombe, 2017; Urbanek et al., 2019), or even the variability in Reconyx devices (Heiniger \& Gillespie, 2018). Other studies have reported a higher performance for Reconyx and Bushnell cameras (Swann et al., 2004; Wellington et al., 2014; Yajima
\& Nakashima, 2021). This inconsistency found in the bibliography could be explained, at least partly, by the target species. Our results showed a significant interaction between camera trap model and species group. The LAC cameras achieved the highest probability of detection for all bird groups, which can be explained by the two levels of Fresnel detection window (Welbourne et al., 2016) that cover a wide range of heights. For those makes with only one horizontal detection band (e.g. RNY), the performance may be lower when detecting small animals (e.g. birds) close to the cameras if the cameras are angled parallel to the slope ground. In the case of SCT camera traps, our results are consistent with those reported in most of the published studies in which Scoutguard models are shown to have a low performance (Driessen et al., 2017; Seccombe, 2017; Swann et al., 2004). In this respect, a relevant problem will emerge in the coming years in those camera trapping long-term studies in which newer cameras usually replace the old (stolen or broken) ones. Overall, our recommendation is to avoid using different camera trap models (and even different models of the same make) in the same study (Fig. 5) or 
considering camera trap model as covariate in the analysis. Additionally, the malfunction observed on Bushnell clock could be a relevant issue for most of camera trap studies in which only one camera is placed per site. This issue should be checked under controlled scenarios since the correction is nontrivial.

The influence of camera trap deployment height on the probability of detection is inconclusive, with some studies suggesting no influence (Jacobs \& Ausband, 2018), lower detection rates for higher deployments (Meek et al., 2016), that small variations in height can result in missed photos (Swann et al., 2004), or that camera traps should be mounted at the target animals' shoulder height (Apps \& McNutt, 2018; Meek et al., 2014). The significant interaction between camera trap deployment height and species group obtained here can be explained by considering the different heights of the species analysed. We set the camera traps at shoulder height for carnivores, big birds, herons and wild boar (Figs. 1-3d). However, all the camera traps were below the horses' shoulders, and above those of small and medium-sized birds, which are the three species groups that achieved the lowest probabilities of detection. These results are particularly relevant for studies that consider more than one target species, such as those focused on estimating species inventories (Tobler et al., 2008) and/or interspecific interactions (Niedballa et al., 2019; TrigueroOcaña et al., 2020). In spite of the fact that camera trapping is usually considered as a multispecies approach (Rovero \& Zimmermann, 2016), the number of detections may be biased (underestimated) for all species for which the camera traps are not set at shoulder height. We recommend multispecies studies using camera trapping exclusively to monitor morphologically similar species or considering a specific sampling design for each species (e.g. placing several camera traps at different heights).

The significant interaction between camera trap deployment height and distance interval suggests that lower deployments increase the probabilities of detection at closer distances, while higher probabilities of detection at further distances are achieved at higher deployments. This result can be explained by the fact that a lower height reduces the chance of small animals passing beneath the camera trap PIR zones at a short distance, but will also reduce the range of distances over which animals can be detected, since vegetation may obstruct the PIR zones (Howe et al., 2017). Furthermore, higher deployments reduce the obstruction of herbaceous vegetation in the PIR zone but increase the probability of small animals passing beneath the PIR zones. We recommend defining the deployment height on the basis of the target species and considering the removal of herbaceous vegetation when small species are the target (Fig. 5). This recommendation is particularly relevant for certain analytical camera trapping methods that assume certain detection close to the camera (Howe et al., 2017).

With regard to the model for trigger speed, we found a strong negative relationship with distance (the closer the animals that entered were to the camera traps, the greater the probability of rapid activation), with the exception of carnivores (Fig. 4a). This may be a consequence of a decrease in
PIR sensitivity as the distance between the animal and the camera trap increases. This decrease is highly noticeable, bearing in mind that for a given speed, the time that the animal spent crossing from one side of the FOV to the other was considerably shorter at the first interval (i.e. from 0 to $2.5 \mathrm{~m}$ from the camera; narrow, and area around $3 \mathrm{~m}^{2}$ ) when compared to the last one (i.e., from 7.5 to $10 \mathrm{~m}$ from the camera; width, and area around $20 \mathrm{~m}^{2}$ ). This result is relevant for camera trapping methods when a specific and rapid detection in the centre of the FOV is assumed (Nakashima et al., 2018), since this should take place only very close to the camera trap (Hofmeester et al., 2017). In the case of carnivores, we suspect that their rapid movement could mask the decrease in PIR sensitivity with distance (Palencia et al., 2021b). With regard to birds, we obtained a significantly higher probability of rapid activation for big than for medium-sized ones, which can be explained by differences in their movement behaviour. While big birds (mostly storks) usually made very slow movements, medium-sized birds (mostly magpies) usually made short flights and nervous movements, thus implying that individuals constantly transited between different distance intervals. In this respect, relatively fast-moving species, such as foxes and magpies require a faster trigger speed than slower species. When carnivores are the target species, and depending on the aim of the study, we recommend using lures to increase the amount of time that animals spend in front of the camera if this does not violate method assumptions. It should be noted that despite the significant effect of the camera trap model, we did not find any association between theoretical trigger speed (ranging from 0.25 to 1.2 , Table 1 ) and the probability of rapid activation. This can be explained as a consequence of the variability in intra-camera trigger times observed in other studies (https:// www.trailcampro.com/collections/trail-camera-reviews; Palencia, Rowcliffe et al., 2021), the type of detection zone (zonal or conical, Driessen et al., 2017), and the relatively wide intervals (width $=2.5 \mathrm{~m}$ ) that have been considered here to evaluate the trigger speed. Future studies therefore should be designed to explore the relevance of the trigger speed by considering shorter intervals, and potentially, not only radials but also angled intervals (Howe et al., 2017; Rowcliffe et al., 2011).

In conclusion, we have described the key factors to consider when working with camera traps, since they determine the performance of cameras. The results reported here provide empirical guidelines for best practices as regards choice of camera trap, set up and study design (Fig. 5), and highlight the high variability of camera trap performance as a consequence of a wide range of factors. Researchers should, therefore, consider the parameters and capabilities of camera traps very carefully in study designs and data analysis, otherwise results may be biased. Moreover, it is highly beneficial for researchers to be aware of the range of cameras available and their relative advantages before investment and use. Empirical experiments, such as those carried out in this study, enhance the applicability, robustness and reliability of camera trapping studies when used to monitor wildlife populations. It will be necessary to constantly re-evaluate the performance of camera traps given the rate at which they continue to evolve. 


\section{Acknowledgements}

The authors would like to thank the DBR wildlife monitoring group and the IREC Game Resources and Wildlife Management group for the loan of some of the camera traps been tested in this work. PP received support from the MINECOUCLM through an FPU grant (FPU16/00039). PA and JV are partly supported by the Spanish Ministry of Economy and Competitiveness (MINECO) research grant (REF: PID2019111699RB-I00).

\section{References}

Akaike, H. (1974). A new look at the statistical model identification. IEEE Transactions on Automatic Control, 19(6), 716-723. https://doi.org/10.1109/TAC.1974.1100705

Apps, P., \& McNutt, J. W. (2018). Are camera traps fit for purpose? A rigorous, reproducible and realistic test of camera trap performance. African Journal of Ecology, 56(4), 710-720. https://doi.org/10.1111/aje.12573

Barasona, J. A., Mulero-Pázmány, M., Acevedo, P., Negro, J. J., Torres, M. J., Gortázar, C., \& Vicente, J. (2014). Unmanned aircraft systems for studying spatial abundance of ungulates: Relevance to spatial epidemiology. PLoS One, 9(12), e115608. https://doi.org/10.1371/journal.pone.0115608.t003

Barroso, P., Barasona, J. A., Acevedo, P., Palencia, P., Carro, F., Negro, J. J., Torres, M. J., Gortázar, C., Soriguer, R. C., \& Vicente, J. (2020). Long-term determinants of tuberculosis in the ungulate host community of doñana national park.

Pathogens, 9(6), 445. https://doi.org/10.3390/

pathogens 9060445

Burton, A. C., Neilson, E., Moreira, D., Ladle, A., Steenweg, R., Fisher, J. T., Bayne, E., \& Boutin, S. (2015). REVIEW: Wildlife camera trapping: A review and recommendations for linking surveys to ecological processes. Journal of Applied Ecology, 52(3), 675-685. http://dx.doi.org/10.1111/1365-2664. 12432

Caravaggi, A., Banks, P. B., Burton, A. C., Finlay, C. M. V., Haswell, P. M., Hayward, M. W., Rowcliffe, M. J., \& Wood, M. D. (2017). A review of camera trapping for conservation behaviour research. Remote Sensing in Ecology and Conservation, 3(3), 109-122. https://doi.org/10.1002/rse2.48

Cusack, J. J., Swanson, A., Coulson, T., Packer, C., Carbone, C., Dickman, A. J., Kosmala, M., Lintott, C., \& Rowcliffe, J. M. (2015). Applying a random encounter model to estimate lion density from camera traps in Serengeti National Park, Tanzania. The Journal of Wildlife Management, 79(6), 10141021. https://doi.org/10.1002/jwmg.902

Delisle, Z. J., Flaherty, E. A., Nobbe, M. R., Wzientek, C. M., \& Swihart, R. K. (2021). Next-generation camera trapping: Systematic review of historic trends suggests keys to expanded research applications in ecology and conservation. Frontiers in Ecology and Evolution, 9, 97. https://doi.org/10. 3389/fevo.2021.617996

Distiller, G. B., Borchers, D. L., Foster, R. J., \& Harmsen, B. J. (2020). Using Continuous-Time Spatial Capture-Recapture models to make inference about animal activity patterns. Ecology and Evolution, 10(20), 11826-11837. https://doi.org/ 10.1002/ece3.6822

Driessen, M. M., Jarman, P. J., Troy, S., \& Callander, S. (2017). Animal detections vary among commonly used camera trap models. Wildlife Research, 44(4), 291. https://doi.org/10.1071/ WR 16228

Edwards, S., Noack, J., Heyns, L., \& Rodenwoldt, D. (2020). Are camera traps a reliable method for estimating activity patterns? A case study comparing technologies for estimating brown hyaena activity curves. Remote Sensing in Ecology and Conservation, 7(2), 129-138. https://doi.org/10.1002/rse2.175

Fancourt, B. A., Sweaney, M., \& Fletcher, D. B. (2018). More haste, less speed: Pilot study suggests camera trap detection zone could be more important than trigger speed to maximise species detections. Australian Mammalogy, 40(1), 118. https:// doi.org/10.1071/AM17004

Findlay, M. A., Briers, R. A., \& White, P. J. C. (2020). Component processes of detection probability in camera-trap studies: Understanding the occurrence of false-negatives. Mammal Research, 65(2), 167-180. http://dx.doi.org/10.1007/ s13364-020-00478-y

Gilbert, N. A., Clare, J. D., Stenglein, J. L., \& Zuckerberg, B. (2021). Abundance estimation of unmarked animals based on camera-trap data. Conservation Biology, 35(1), 88-100. https:// doi.org/10.1111/cobi.13517

Glover-Kapfer, P., Soto-Navarro, C. A., \& Wearn, O. R. (2019). Camera-trapping version 3.0: Current constraints and future priorities for development. Remote Sensing in Ecology and Conservation, 5(3), 209-223. https://doi.org/10.1002/rse2.106

Guillera-Arroita, G., Ridout, M. S., \& Morgan, B. J. (2010). Design of occupancy studies with imperfect detection. Methods in Ecology and Evolution, 1(2), 131-139. https://doi. org/10.1111/j.2041-210X.2010.00017.x

Harrison, X. A., Donaldson, L., Correa-Cano, M. E., Evans, J., Fisher, D. N., Goodwin, C. E. D., Robinson, B. S., Hodgson, D. J., \& Inger, R. (2018). A brief introduction to mixed effects modelling and multi-model inference in ecology. PeerJ, 6, e4794. https://doi.org/10.7717/peerj.4794

Heiniger, J., \& Gillespie, G. (2018). High variation in camera trap-model sensitivity for surveying mammal species in northern Australia. Wildlife Research, 45(7), 578. https://doi. org/10.1071/WR18078

Hofmeester, T. R., Cromsigt, J. P., Odden, J., Andrén, H., Kindberg, J., \& Linnell, J. D. (2019). Framing pictures: A conceptual framework to identify and correct for biases in detection probability of camera traps enabling multi-species comparison. Ecology and Evolution, 9(4), 2320-2336. https:// doi.org/10.1002/ece3.4878

Hofmeester, T. R., Rowcliffe, J. M., \& Jansen, P. A. (2017). A simple method for estimating the effective detection distance of camera traps. Remote Sensing in Ecology and Conservation, 3(2), 81-89. https://doi.org/10.1002/rse2.25

Hofmeester, T. R., Thorsen, N. H., Cromsigt, J. P., Kindberg, J., Andrén, H., Linnell, J. D., \& Odden, J. (2021). Effects of 
camera-trap placement and number on detection of members of a mammalian assemblage. Ecosphere, 12(7), e03662. https://doi.org/10.1002/ecs2.3662

Howe, E. J., Buckland, S. T., Després-Einspenner, M.-L., \& Kühl, H. S. (2017). Distance sampling with camera traps. Methods in Ecology and Evolution, 8(11), 1558-1565. https:// doi.org/10.1111/2041-210X.12790

Hughson, D., Darby, N., \& Dungan, J. (2010). Comparison of motion-activated cameras for wildlife investigations. California Fish and Game, 96(2), 101-109. https://www.researchgate.net/ publication/288112505_Comparison_of_motion-activated_ cameras_for_wildlife_investigations

Jacobs, C. E., \& Ausband, D. E. (2018). An evaluation of camera trap performance - What are we missing and does deployment height matter? Remote Sensing in Ecology and Conservation, 4(4), 352-360. https://doi.org/10.1002/rse2.81

Kays, R., Hody, A., Jachowski, D. S., \& Parsons, A. W. (2021). Empirical evaluation of the spatial scale and detection process of camera trap surveys. Movement Ecology, 9(1), 1-13. https:// doi.org/10.1186/s40462-021-00277-3

Kays, R., Tilak, S., Kranstauber, B., Jansen, P. A., Carbone, C., Rowcliffe, M. J., Fountain, T., Eggert, J., \& He, Z. (2010). Monitoring wild animal communities with arrays of motion sensitive camera traps. arXiv preprint arXiv:1009.5718. http:// dblp.uni-trier.de/db/journals/corr/corr1009.html\#abs-1009-5718

McIntyre, T., Majelantle, T. L., Slip, D. J., \& Harcourt, R. G. (2020). Quantifying imperfect camera-trap detection probabilities: Implications for density modelling. Wildlife Research, 47(2), 177. https://doi.org/10.1071/WR19040

Meek, P. D., Ballard, G., Claridge, A., Kays, R., Moseby, K., O’Brien, T., O'Connell, A., Sanderson, J., Swann, D. E., Tobler, M., \& Townsend, S. (2014). Recommended guiding principles for reporting on camera trapping research. Biodiversity and Conservation, 23, 2321-2343. https://doi.org/ 10.1007/s10531-014-0712-8

Meek, P. D., Ballard, G. A., \& Falzon, G. (2016). The higher you go the less you will know: placing camera traps high to avoid theft will affect detection. Remote Sensing in Ecology and Conservation, 2(4), 204-211. https://doi.org/10.1002/rse2.28

Meek, P. D., Ballard, G.-A., \& Fleming, P. J. S. (2015). The pitfalls of wildlife camera trapping as a survey tool in Australia. Australian Mammalogy, 37(1), 13. https://doi.org/10. 1071/AM14023

Meek, P. D., \& Pittet, A. (2012). User-based design specifications for the ultimate camera trap for wildlife research. Wildlife Research, 39(8), 649. https://doi.org/10.1071/ WR12138

Mills, K. L., Harissou, Y., Gnoumou, I. T., Abdel-Nasser, Y. I., Doamba, B., \& Harris, N. C. (2020). Comparable space use by lions between hunting concessions and national parks in West Africa. Journal of Applied Ecology, 57(5), 975-984.

Nakashima, Y., Fukasawa, K., \& Samejima, H. (2018). Estimating animal density without individual recognition using information derivable exclusively from camera traps. Journal of Applied Ecology, 55(2), 735-744. https://doi.org/10.1111/ 1365-2664.13059

Newey, S., Davidson, P., Nazir, S., Fairhurst, G., Verdicchio, F., Irvine, R. J., \& van der Wal, R. (2015). Limitations of recreational camera traps for wildlife management and conservation research: A practitioner's perspective. Ambio, 44 (S4), 624-635. https://doi.org/10.1007/s13280-015-0713-1

Niedballa, J., Wilting, A., Sollmann, R., Hofer, H., \& Courtiol, A. (2019). Assessing analytical methods for detecting spatiotemporal interactions between species from camera trapping data. Remote Sensing in Ecology and Conservation, 5 (3), 272-285. https://doi.org/10.1002/rse2.107

Palencia, P., Fernández-López, J., Vicente, J., \& Acevedo, P. (2021). Innovations in movement and behavioural ecology from camera traps: Day range as model parameter. Methods in Ecology and Evolution, 12(7), 1201-1212. https://doi.org/10. 1111/2041-210X.13609

Palencia, P., Rowcliffe, J. M., Vicente, J., \& Acevedo, P. (2021). Assessing the camera trap methodologies used to estimate density of unmarked populations. Journal of Applied Ecology, 58(8), 1583-1592. https://doi.org/10.1111/1365-2664.13913

Rovero, F., \& Zimmermann, F. (2016). Camera Trapping for Wildlife Research (Pelagic Pu).

Rowcliffe, J. M., Carbone, C., Jansen, P. A., Kays, R., \& Kranstauber, B. (2011). Quantifying the sensitivity of camera traps: An adapted distance sampling approach. Methods in Ecology and Evolution, 2(5), 464-476. https://doi.org/10.1111/ j.2041-210X.2011.00094.X

Rowcliffe, J. M., Kays, R., Kranstauber, B., Carbone, C., \& Jansen, P. A. (2014). Quantifying levels of animal activity using camera trap data. Methods in Ecology and Evolution, 5 (11), 1170-1179. https://doi.org/10.1111/2041-210x.12278

Seccombe, S. (2017). ZSL trail camera comparison testing. https://www.wildlabs.net/sites/default/files/community/files/zsl_ trail_camera_comparison_for_external_use.pdf

Swann, D. E., Hass, C. C., Dalton, D. C., \& Wolf, S. A. (2004). Infrared-triggered cameras for detecting wildlife: an evaluation and review. Wildlife Society Bulletin, 32(2), 357-365. https:// doi.org/10.2193/0091-7648(2004)32[357:ICFDWA]2.0.CO

Tobler, M. W., Carrillo-Percastegui, S. E., Leite Pitman, R., Mares, R., \& Powell, G. (2008). An evaluation of camera traps for inventorying large- and medium-sized terrestrial rainforest mammals. Animal Conservation, 11(3), 169-178. https://doi.org/10.1111/j.1469-1795.2008.00169.x

Triguero-Ocaña, R., Vicente, J., Palencia, P., Laguna, E., \& Acevedo, P. (2020). Quantifying wildlife-livestock interactions and their spatio-temporal patterns: Is regular grid camera trapping a suitable approach? Ecological Indicators, 117, 106565. https://doi.org/10.1016/j.ecolind.2020.106565

Urbanek, R. E., Ferreira, H. J., Olfenbuttel, C., Dukes, C. G., \& Albers, G. (2019). See what you've been missing: An assessment of Reconyx ${ }^{\circledR}$ PC900 Hyperfire cameras. Wildlife Society Bulletin, 43(4), 630-638. https://doi.org/10.1002/wsb. 1015 
Welbourne, D. J., Claridge, A. W., Paull, D. J., \& Lambert, A. (2016). How do passive infrared triggered camera traps operate and why does it matter? Breaking down common misconceptions. Remote Sensing in Ecology and Conservation, 2(2), 77-83. https://doi.org/10.1002/rse2.20

Wellington, K., Bottom, C., Merrill, C., \& Litvaitis, J. A. (2014). Identifying performance differences among trail cameras used to monitor forest mammals. Wildlife Society Bulletin, 38(3), 634-638. https://doi.org/10.1002/wsb.425

Yajima, G., \& Nakashima, Y. (2021). Can video traps reliably detect animals? Implications for the density estimation of animals without individual recognition. Mammal Study, 46(3), 1-7. https://doi.org/10.3106/ms2020-0055

Zuur, A. F., Ieno, E. N., \& Elphick, C. S. (2010). A protocol for data exploration to avoid common statistical problems.
Methods in Ecology and Evolution, 1(1), 3-14. https://doi.org/ 10.1111/j.2041-210X.2009.00001.x

\section{Supporting Information}

Additional Supporting Information may be found in the online version of this article:

Appendix S1. Video clip of a red deer entering the field of view of the camera traps.

Appendix S2. Species grouping details.

Appendix S3. Tables showing best models for probability of detection and trigger speed.

Appendix S4. Time delay observed for Bushnell camera traps. 